\title{
Differences in forward angular light scattering distributions between M1 and M2 macrophages
}

David L. Halaney

Aydin Zahedivash

Jennifer E. Phipps

Tianyi Wang

Jordan Dwelle

Claude Jourdan Le Saux

Reto Asmis

Thomas E. Milner

Marc D. Feldman 


\title{
Differences in forward angular light scattering distributions between M1 and M2 macrophages
}

\author{
David L. Halaney, ${ }^{\mathrm{a}, \mathrm{b}}$ Aydin Zahedivash, ${ }^{\mathrm{c}}$ Jennifer E. Phipps, ${ }^{\mathrm{a}}$ Tianyi Wang, ${ }^{\mathrm{c}}$ Jordan Dwelle, ${ }^{\mathrm{b}, \mathrm{c}}$ \\ Claude Jourdan Le Saux, ${ }^{a}$ Reto Asmis, ${ }^{d}$ Thomas E. Milner, ${ }^{c}$ and Marc D. Feldman ${ }^{\mathrm{a}, \mathrm{b}, *}$ \\ aniversity of Texas Health Science Center at San Antonio, Division of Cardiology, Department of Medicine, 7703 Floyd Curl Drive, San Antonio, \\ Texas 78229, United States \\ bSouth Texas Veterans Health Care System, Department of Veterans Affairs, 7400 Merton Minter, San Antonio, Texas 78229, United States \\ 'University of Texas at Austin, Department of Biomedical Engineering, 1 University Station C0800, Austin, Texas 78712, United States \\ dUniversity of Texas Health Science Center at San Antonio, Departments of Clinical Laboratory Sciences and Biochemistry, 7703 Floyd Curl Drive, \\ San Antonio, Texas 78229, United States
}

\begin{abstract}
The ability to distinguish macrophage subtypes noninvasively could have diagnostic potential in cancer, atherosclerosis, and diabetes, where polarized M1 and M2 macrophages play critical and often opposing roles. Current methods to distinguish macrophage subtypes rely on tissue biopsy. Optical imaging techniques based on light scattering are of interest as they can be translated into biopsy-free strategies. Because mitochondria are relatively strong subcellular light scattering centers, and M2 macrophages are known to have enhanced mitochondrial biogenesis compared to M1, we hypothesized that M1 and M2 macrophages may have different angular light scattering profiles. To test this, we developed an in vitro angle-resolved forward light scattering measurement system. We found that M1 and M2 macrophage monolayers scatter relatively unequal amounts of light in the forward direction between 1.6 deg and 3.2 deg with M2 forward scattering significantly more light than $\mathrm{M} 1$ at increasing angles. The ratio of forward scattering can be used to identify the polarization state of macrophage populations in culture. ๑ 2015 Society of Photo-Optical Instrumentation Engineers (SPIE) [DOI: 10.1117/1.JBO.20.11.115002]
\end{abstract}

Keywords: macrophage; M1 M2; angular light scattering.

Paper 150215RR received Mar. 31, 2015; accepted for publication Oct. 9, 2015; published online Nov. 5, 2015.

\section{Introduction}

Macrophages are widely distributed immune cells, which play critical roles in homeostasis and disease states. ${ }^{1-3}$ Macrophages are highly versatile and plastic, and can be phenotypically polarized to express different functional programs in response to microenvironmental signals. ${ }^{1-6}$ While it is generally believed that macrophages represent a continuum of functional states rather than discrete stable subpopulations, the extreme ends of the continuum can be broadly classified into two main groups: the classically activated (M1) macrophages and the alternatively activated (M2) macrophages. ${ }^{1-4,7}$ M1 macrophages are proinflammatory, exhibiting potent antimicrobial properties through production of nitric oxide and radical oxygen intermediates, while M2 macrophages are anti-inflammatory, with important roles in wound healing and fibrosis. ${ }^{1-4,7}$ Macrophages are believed to be drivers or facilitators of many common diseases. ${ }^{1,3,8}$ In cancer, a tumor-killing role has been described for M1 macrophages. ${ }^{1,3,9}$ However, tumor-associated macrophages are phenotypically and functionally similar to M2 macrophages, playing a detrimental protumoral role. ${ }^{1-4,7-9}$ In obesity-induced type- 2 diabetes, adipose tissue macrophages switch from an M2-like phenotype, which maintains insulin sensitivity and glucose tolerance, to an M1like phenotype, which contributes to the development of insulin resistance. ${ }^{1,3,8-11}$ Finally, in experimental atherosclerosis, a shift from the M2 to the M1 phenotype can signify the progression

*Address all correspondence to: Marc D. Feldman, E-mail: feldmanm @ uthscsa .edu from stable to unstable lesions. ${ }^{10} \mathrm{M} 2$ macrophages, on the other hand, produce cytokines with antiatherogenic and profibrotic properties, which promote plaque stability. ${ }^{3,8}$ The ability to detect subpopulations of M1 and M2 macrophages in atherosclerotic plaques may be feasible, as several investigators have now reported distinct localization of M1 and M2 macrophages to discrete zones of the atherosclerotic plaque..$^{5,6,12}$

Identification of macrophage subtypes in vivo is complicated, as markers (genes, proteins, or metabolic products) associated with M1 or M2 macrophages are usually more abundant in, but rarely specific for, a particular subtype. ${ }^{3}$ Accordingly, distinguishing between M1 and M2 macrophage subtypes in vitro/ ex vivo is achieved through characterization of gene expression profiles (microarrays ${ }^{12}$ or qPCR of multiple genes ${ }^{5,6}$ ), functional assays of nitric oxide (M1) or arginase 1 (M2) production ${ }^{9}$ or other cytokines, ${ }^{5}$ immunohistochemical labeling of surface receptors, ${ }^{5,6,12,13}$ and flow cytometry. ${ }^{14}$ However, these techniques cannot currently be used clinically for in vivo tissue diagnostics (e.g., cancer, diabetes, or atherosclerosis) without tissue biopsy. Biopsy-free, label-free techniques that have the potential to distinguish M1 and M2 macrophages in vivo in patients are of interest.

Because light has a wavelength $(1 \mu \mathrm{m})$ that is on the same order as the size of cellular organelles, optical approaches to distinguish between different cell types have been studied. In fact, some of the first reported cellular light scattering studies

1083-3668/2015/\$25.00 @ 2015 SPIE 
were completed by Chance, Tedeschi, and Packer, who observed changes in light scattering due to cellular metabolism. These studies eventually led to investigation on the mitochondria as the source of some of the observed changes. ${ }^{15-20}$ In particular, angle-resolved light scattering techniques have been used to identify differences in the distribution, size, shape, and relative number of cells and their intracellular organelles. ${ }^{21-26}$ Many of these intracellular structural differences have been linked to functional processes such as apoptosis, ${ }^{27}$ mitochondrial fragmentation, ${ }^{26}$ rounding, ${ }^{21}$ swelling, ${ }^{22,23}$ alterations in lysosomal structure, ${ }^{28}$ and changes in nuclear size. ${ }^{29}$ For example, Pyhtila et al. ${ }^{29}$ have used a light scattering technique to identify increased nuclear size of epithelial cells in the esophagus to identify precancerous lesions. Imaging techniques that distinguish light scattering angle are of interest as they can potentially be translated into biopsyfree, label-free strategies.

M1 and M2 macrophages have known differences in intracellular organelles, with M2 having greater mitochondrial density than M1. ${ }^{30-32}$ Since mitochondria are known to be relatively strong subcellular light scattering centers, ${ }^{21,23-25}$ differences in mitochondrial density are expected to cause differences in the angular distribution of light scattering intensities between the two macrophage subtypes. Therefore, we hypothesize that M1 and M2 macrophages will differ in their angular distributions of light scattering and that these differences may be used to distinguish these two subtypes in culture. To test this hypothesis, we constructed an optical system to measure the forward light scattering angular distributions of murine M1 and M2 macrophage monolayers in vitro. Although backscatter measurements are more relevant to bulk tissues, forward scattering measurements are more appropriate when studying cell cultures because signal intensity is greater than in backscattering instrumentation, ${ }^{33}$ and a forward scattering measurement is simpler and avoids specular reflections from the culture dish.

\section{Methods}

\subsection{Instrumentation}

The experimental setup was designed to measure the intensity of forward scattered light as a function of angle from macrophage cultures. A superluminescent diode (Superlum: SLD-381-MP3DIL-SM) with a center wavelength of $942.8 \mathrm{~nm}$ and FWHM spectral width of $77.1 \mathrm{~nm}$ was powered by an Agilent E3615A power supply (8.99 V, $0.35 \mathrm{~A})$ and operated at an emission intensity of $0.5 \mathrm{~mW}$. Light emitted from the SLD was coupled into a single mode optical fiber, collimated to a 3$\mathrm{mm}$ diameter (collimation angle $=0.012 \mathrm{deg}$ ), and reflected from a mirror and directed at normal incidence onto a macrophage culture. The macrophage culture was positioned in the front focal plane of a 40-mm diameter, 50.8-mm focal length Steinheil triplet lens (JML). Behind the lens, a Fujifilm X-A1 digital camera (APS-C) with a $23.6 \mathrm{~mm} \times 15.6 \mathrm{~mm}$ CMOS sensor (16.5 megapixel) was positioned so that the sensor was in the back focal plane (Fig. 1). By positioning the macrophage culture in the front focal plane and the CMOS sensor in the back focal plane, the CMOS sensor recorded a two-dimensional map of the angular distribution of forward scattered light intensity from the macrophage cells. The angular resolution of the system was limited by the pixel spacing on the CMOS sensor and was $0.005 \mathrm{deg}$. The incident beam was not blocked, and pixels near the center of the scattering image which received the signal from the incident beam were removed prior to analysis, as discussed below.

\subsection{Mouse Husbandry}

Macrophage cultures were obtained from 2- to 4-month-old male mice (C57BL/6 wild type mice from Charles River Laboratories). All experimental procedures and humane care

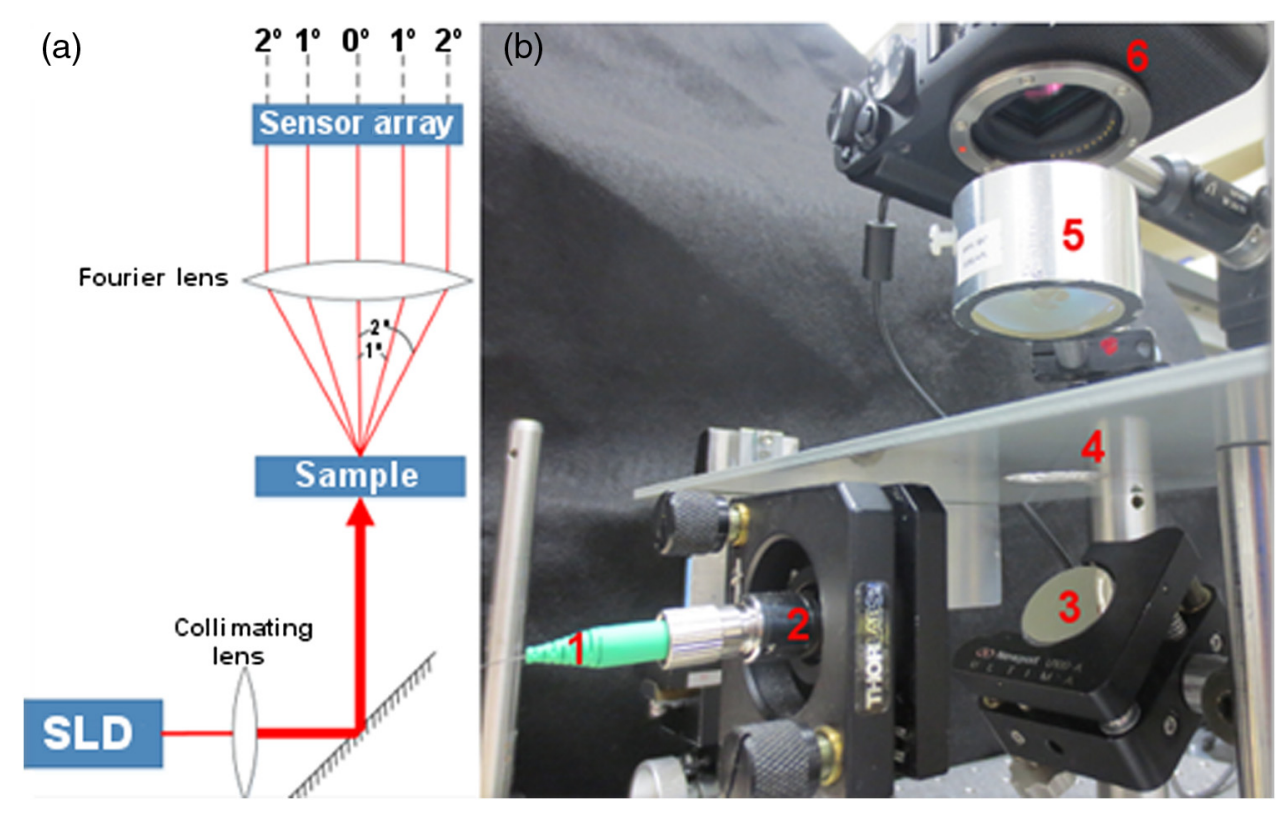

Fig. 1 Forward scattering angle imager. (a) Illustration of scattering-angle imaging system. (b) Scattering-angle imaging system; 1-SLD illumination via an optical fiber, 2-collimator lens to broaden incident beam and provide increased angular range on the CMOS sensor, 3-mirror, 4-sample (macrophage culture), 5-50.8 mm focal length collimator lens, and 6-CMOS sensor array (inside digital camera). 
of the mice were conducted in compliance with the University of Texas Health Science Center Institutional Animal Care and Use Committee approval.

\subsection{Macrophage Cell Cultures}

Murine peritoneal immune cells were harvested from euthanized mice by peritoneal lavage. Briefly, $10 \mathrm{ml}$ of culture media [ $47 \%$ v/v RPMI 1640 (Gibco 21870), 47\% v/v RPMI 1640 (Cellgro 10-043-CV), $1 \%$ v/v nonessential amino acids $10 \mathrm{mM}$ (Gibco 11140-050), 1\% v/v Glutamax-I 200 mM (Gibco 35050-061), $1 \% \mathrm{v} / \mathrm{v}$ sodium pyruvate $100 \mathrm{mM}$ (Gibco 11360-070), 1\% v/v Penicillin-Streptomycin (Gibco 15140-122), and 2\% v/v N-(2hydroxyethyl)piperazine- $N^{\prime}$-(2-ethanesulfonic acid) $1 \mathrm{M}$ (HEPES; Sigma)] with $2 \%$ fetal bovine serum (FBS; Gibco 10082-147) was injected into the peritoneal cavity, the mouse was gently shaken to dislodge peritoneal cells, and the media were recovered. The peritoneal cells were concentrated into a pellet by centrifugation $(400 \mathrm{~g})$ and resuspended in $1 \mathrm{ml}$ of culture media. A dilution of this cell suspension was counted using a hemacytometer to determine the number of immune cells recovered. Of this number, approximately 50\% are macrophages, and the majority of the remaining cells are T- and Blymphocytes. To purify the cell suspension to primarily macrophages ( $\sim 95 \%$ macrophages, with the remaining $5 \%$ representing primarily neutrophils), T- and B-lymphocytes were removed from the suspension using magnetic Dynabeads conjugated to antibodies against T- (Life Technologies 114-43D) and B-lymphocytes (Life Technologies 114-41D). The resulting cell suspension (95\% macrophages) was counted again using a hemacytometer, and the suspension was adjusted appropriately to plate 250,000 macrophages per well in a 24-well cell culture plate (BD Falcon 08-772-1) in $0.5 \mathrm{ml}$ of cell culture media with $10 \%$ FBS.

The cultures were incubated overnight $\left(37^{\circ} \mathrm{C}, 5 \% \mathrm{CO}_{2}, 90 \%\right.$ humidity) to achieve adhesion of the macrophages to the well surface in a monolayer of near confluence. The media were then replaced with $0.5 \mathrm{ml}$ fresh macrophage culture media $\left(37^{\circ} \mathrm{C}\right)$ with $10 \%$ FBS supplemented with cytokines. M1 culture media were supplemented with $10 \mathrm{ng} / \mathrm{ml}$ lipopolysaccharide (LPS; Calbiochem 437620-5MG) and $50 \mathrm{ng} / \mathrm{ml}$ interferongamma (IFN- $\gamma$; PeproTech 315-05-20UG). M2 culture media were supplemented with $10 \mathrm{ng} / \mathrm{ml}$ interleukin-4 (IL-4; PeproTech 214-14-20UG). Resting macrophage culture media were not supplemented with cytokines, and these cultures were used as controls to verify the molecular/functional phenotype of M1 and M2 macrophages. Cultures were incubated with cytokines for $24 \mathrm{~h}$ before optical measurement.

\subsection{Mathematical Background}

In this study, we measured differences in forward-directed scattering between M1 and M2 macrophages. The angle-resolved scattering pattern is projected onto the image plane of the Fourier lens according to the following equation:

$u\left(x^{\prime}, y^{\prime}\right)=\frac{A_{0} \mathrm{e}^{j 2 k f}}{j \lambda f} B_{l} A_{C}\left(\frac{x^{\prime}}{\lambda f}, \frac{y^{\prime}}{\lambda f}\right)$,

where $x^{\prime}$ and $y^{\prime}$ are the spatial coordinates on the CMOS sensor (imaging plane), $f$ is the lens focal distance, $\lambda$ is the vacuum wavelength of the source, $k$ is $2 \pi / \lambda, B_{l}$ is the optical thickness of the lens, $A_{0}$ is the magnitude and absolute phase of the incident wave field, and $A_{C}$ is the Fourier transform of the amplitude of the wave field emerging from the cell layer, which is influenced by the refractive index of the scatterers $\left(n=1.33 \text { for media }^{34} \text { and } n=1.36 \text { for cytoplasm }{ }^{35}\right)_{\text {involved. }}{ }^{36}$ The CMOS sensor records this information convolved with the pupil's impulse response function. Because the pupil was large, we take the impulse function to be a delta function. At lateral position $\left(x^{\prime}, y^{\prime}\right)$ on the CMOS sensor, the corresponding angle $(\alpha)$ of the forward scattered light can be written as

$\alpha=\tan ^{-1}\left(\frac{\sqrt{x^{\prime 2}+y^{\prime 2}}}{f}\right)$.

This result demonstrates that the observed angle-resolved intensity is dependent on the interactions with the cell layer and other experimental parameters.

\subsection{Scattering Angle Imaging}

Following the $24 \mathrm{~h}$ cytokine treatment, the cultures were changed to fresh media without $\mathrm{FBS}\left(37^{\circ} \mathrm{C}\right)$ and imaged on an inverted microscope to document the baseline appearance, and then light scattering angle images were recorded with $1 \mathrm{~s}$ integration time. To reduce the effects of ambient light, the imaging system was covered with thick black felt and the room lights were turned off during imaging. Following scattering angle imaging, the cells were assessed microscopically to confirm that the morphology did not change during imaging. Figure 2 shows the typical cultures of M1 and M2 macrophages.

\subsection{Analysis of Scattering Data}

Forward scattered light intensity data were recorded by the CMOS sensor from 0 deg to $8.8 \mathrm{deg}$ for M1 and M2 cell cultures. Only forward scattered light intensity data at angles between $1.6 \mathrm{deg}$ and $3.2 \mathrm{deg}$ were analyzed. At angles less than $1.6 \mathrm{deg}$, nonscattered collimated light focused on the CMOS sensor resulted in an offset that precluded inclusion of this data in the analysis. Control images without cells confirmed that there was no incident blooming by $1.6 \mathrm{deg}$. At angles larger than $3.2 \mathrm{deg}$, the signal intensity was substantially reduced, and signal-to-noise ratio was less than unity, so these data were not included in the analysis. We imaged and analyzed $n=12 \mathrm{M} 1$ cultures, $n=10$ M2 cultures, and $n=4$ controls (media in wells without cells) over the course of four independent experiments, each conducted 1 week apart. For our analysis, each light scattering image was sampled up to 24 times by measuring the intensity versus the distance from the center of the scattering image $(0 \mathrm{deg})$ along a radial line every $15 \mathrm{deg}$ using MATLAB. Artifacts such as double-light reflections from the cell culture plate were identified, and radial sampling lines effected by these artifacts were excluded from analysis. We azimuthally averaged the radial sampling lines for each scattering image to determine an average intensity profile of light scattering as a function of angle for each culture. Moreover, our results are independent of cell orientation due to the implicit averaging of thousands of cells being imaged simultaneously. The average scattering angle profile of the $n=4$ control cultures without cells was subtracted from the scattering profile of each culture to remove the background due to the cell culture plate and media. 


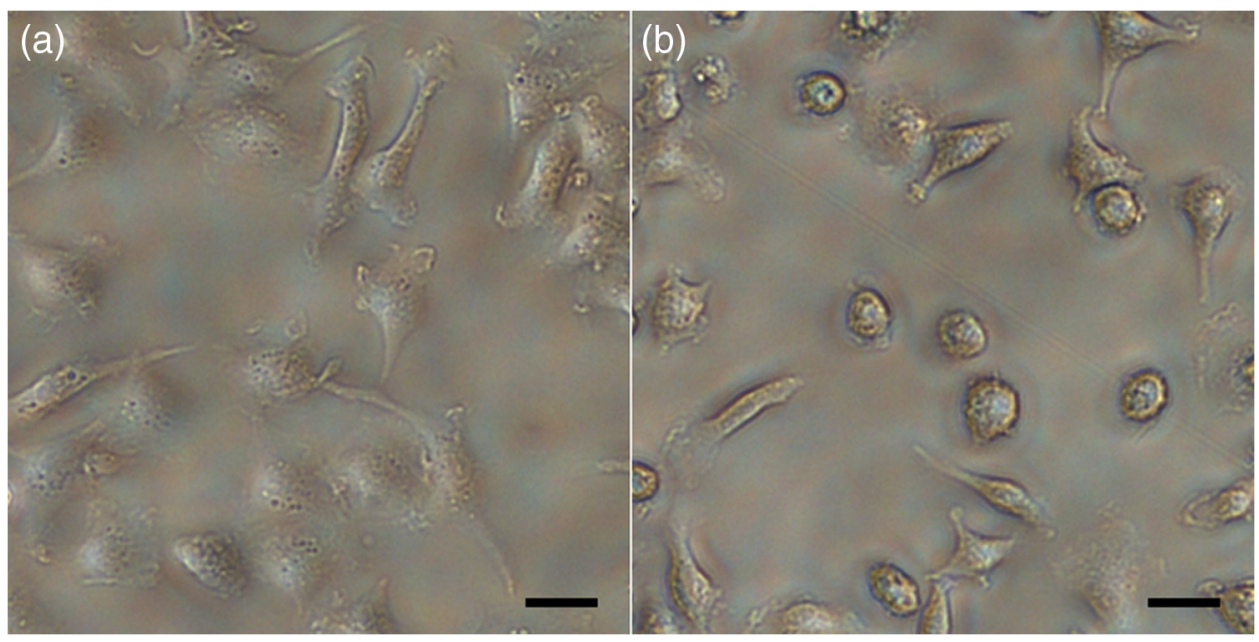

Fig. 2 Typical cell cultures. (a) M1 have a broad and flat phenotype and are semitransparent, (b) M2 have a round phenotype with granular cytoplasm and are opaque. Scale bars $=20 \mu \mathrm{m}$.

\subsection{Molecular/Functional Assays to Confirm the Identity of M1 and M2 Macrophages}

Additional macrophage cultures were prepared in an identical manner and used to confirm polarization of macrophages into M1 and M2 subtypes. Each assay was run in triplicate in three independent experiments.

\subsubsection{Quantitative reverse transcription polymerase chain reaction}

Total RNA was extracted from macrophages immediately following the $24 \mathrm{~h}$ cytokine treatment using the RNeasy ${ }^{\mathrm{TM}}$ kit (Qiagen Inc., Valencia, Virginia). Equal quantities of RNA (200 ng) were converted into first-strand cDNA using random hexamers (SuperScript III First-Strand Synthesis System for RTPCR $^{\mathrm{TM}}$, Invitrogen Life Technologies, Grand Island, New York). The mRNA abundance was analyzed by RT-PCR (qPCR) using commercially available TaqMan probes (Applied Biosystems, Carlsbard, California). Results were analyzed by the $\Delta \Delta C_{t}$ method $^{37}$ with $18 \mathrm{~s}$ rRNA as the internal control.

\subsubsection{Nitric oxide functional assay (M1)}

A colorimetric ELISA, nitric oxide, or Griess reagent system assay was used on the plated macrophage cells (Promega G2930, Madison, Wisconsin) according to the manufacturer's instructions. The OD of each sample was read at $540 \mathrm{~nm}$ and compared to a standard curve of known nitrite concentration.

\subsubsection{Arginase functional assay (M2)}

Arginase activity was quantified by measuring the conversion of L-arginine into urea as previously reported. ${ }^{38}$ In brief, $10 \mathrm{mM}$ $\mathrm{MnCl}_{2}$ in $50 \mathrm{mM}$ Tris- $\mathrm{HCl}$ was added to the lysed sample, and the mixture was then incubated at $56^{\circ} \mathrm{C}$ to activate the arginase enzyme. L-Arginine was then added, and the samples were incubated at $37^{\circ} \mathrm{C}$ for $2 \mathrm{~h}$ and terminated by the addition of an acid solution. The colorimetric indicator, $6 \% \alpha$-isonitrosopropiophenone in $100 \%$ ethanol, was then added to each tube and heated to $95^{\circ} \mathrm{C}$, and the $\mathrm{OD}$ of each sample was read at $540 \mathrm{~nm}$. Arginase activity in the samples was determined based on the standard curve of known urea concentration.

\subsection{Data Analysis and Statistics}

All statistical analyses were performed in MATLAB R2013b. $T$-tests were used for genetic assays and for the comparison of ratios of scattered light intensity (1.6 deg:3.2 deg) between M1 and M2 cultures. One-way ANOVA with post-hoc Tukey testing was used for comparison of ratios of scattered light intensity (1.6 deg:3.2 deg) within subtypes and between independent experiments. A $p$-value less than 0.05 was considered significant. Values are provided as mean \pm standard deviation.

\section{Results}

Mean M1 and M2 scattering angle profiles demonstrate a clear separation for forward scattering angles analyzed [Fig. 3(a)], with M2 forward scattering relatively more light than M1 at increased angles. At $1.6 \mathrm{deg}$, the two macrophage subtypes scattered similar amounts of light, with the ratio of M2 scattering intensity to M1 scattering intensity approximately unity. This ratio increased in a nearly linear manner to the largest scattering angle analyzed [3.2 deg, Fig. 3(b)].

Due to the different rates of signal drop-off between M1 and M2 cultures at increased scattering angles and because the signal intensity from M1 cultures decreased faster than M2 cultures, we compared the data at the ends of our measured range. The ratio of scattered light intensity at $1.6 \mathrm{deg}$ to intensity at $3.2 \mathrm{deg}$ was found to distinguish M1 $(4.31 \pm 0.65)$ from M2 ( $2.75 \pm 0.48)$ cultures; $p<0.001$. Since our data were collected over the course of four independent experiments, we searched for statistical variation of this ratio between experiments using one-way ANOVA with post-hoc Tukey testing for multiple comparisons; M1 $p=0.134$ (ANOVA) with all pairwise comparisons nonsignificant (Tukey); and M2 $p=0.114$ (ANOVA) with all pairwise comparisons nonsignificant (Tukey). This ratio was found to consistently distinguish the two subtypes between the four independent experiments.

To confirm successful polarization of macrophages into M1 or M2 phenotypes after cytokine treatment, we performed molecular and functional assays with unpolarized resting macrophage cultures as controls. Media from macrophage cultures treated with LPS and IFN- $\gamma$ contained significantly higher nitric oxide concentration than media control resting macrophage cultures after $24 \mathrm{~h}(p=0.05)$, consistent with the functional 

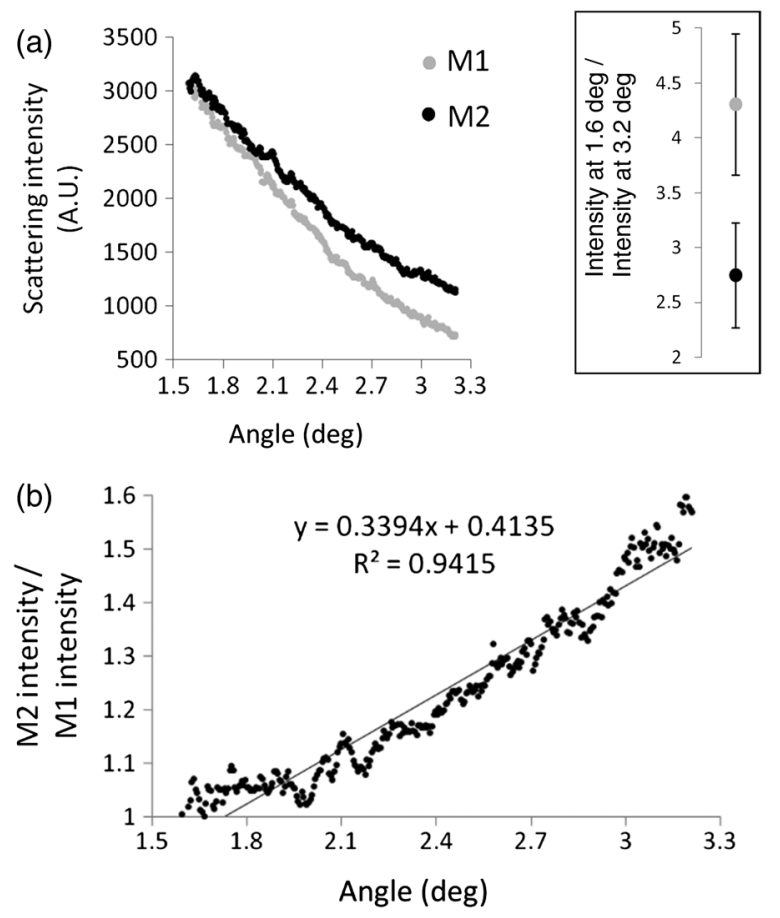

Fig. 3 Differences in angular light scattering distributions between $\mathrm{M} 1$ $(n=12)$ and M2 $(n=10)$ macrophage cultures. (a) After subtracting the background from each scattering image, mean M1 and M2 scattering intensities are plotted against scattering angle (deg). The mean ratio of scattering intensity between $1.6 \mathrm{deg}$ and $3.2 \mathrm{deg}$, the extreme ends of our measured range and where intensity difference was greatest, was significantly greater than for M1 than M2 macrophages $(p<0.001)$ (see inset; error bars represent the standard deviation). (b) The mean M2 scattering profile from (a) was divided by the mean M1 scattering profile, demonstrating that both subtypes scatter similar amounts of light at 1.6 deg with a ratio $\sim 1$, and this ratio increases in a nearly linear fashion to 1.6 at a scattering angle of 3.2 deg.

activities of the M1 phenotype [Fig. 4(a)]. Similarly, media from macrophage cultures treated with IL-4 contained significantly higher arginase 1 concentrations than media from control resting macrophage cultures after $24 \mathrm{~h}(p=0.0074)$, consistent with the functional activities of the M2 phenotype [Fig. 4(b)].

The mRNA profile obtained from the LPS- and IFN- $\gamma$-treated macrophages revealed significantly enhanced expression of Arg2 mRNA $(p=0.0286)$ and TNF- $\alpha$ mRNA $(p=0.0110)$ compared to untreated macrophages [Figs. 4(c) and 4(e)], consistent with an M1 expression profile. Similarly, IL-4-treated macrophages showed a significant increase in the expression of Arg1 mRNA $(p=0.0010)$ and Ym1 mRNA ( $p=0.0454)$ [Figs. 4(d) and 4(f)] compared to untreated macrophages, consistent with an M2 expression profile. Taken together, these complementary functional and molecular assays indicate successful polarization of macrophages into M1 and M2 phenotypes.

\section{Discussion}

In this study, we sought to test the hypothesis that M1 and M2 macrophages differ in their refractive index distributions and thus their angular distribution of forward scattered light profiles. We constructed an optical instrument to examine M1 and M2 cultures in vitro and measure the angular distribution of forward scattered light. Our results show that M2 macrophages scatter more light than M1 macrophages in the angular range of
$1.6 \mathrm{deg}$ to $3.2 \mathrm{deg}$, and that the rate of signal drop-off with increased scattering angle was steeper for M1 macrophages. The result is a significantly higher ratio of forward scattering intensity for M1 compared to M2 macrophage cultures. Finally, we performed molecular and functional assays of M1, M2, and resting (control) macrophage cultures to confirm successful polarization of macrophages following cytokine treatment.

From flow cytometry, forward light scattering at small angles ( $0.5 \mathrm{deg}$ to $1.5 \mathrm{deg}$ ) is predominantly due to the whole-cell volume. ${ }^{23,24}$ In our study, M1 macrophages in culture appeared on average larger, flatter, and more optically transparent compared to M2 macrophages in culture. M2 macrophages appeared smaller, more spherical, and more optically opaque (Fig. 2). In flow cytometry, where the beam interacts with one cell at a time, the membrane defines the whole cell as a scattering object. However, in our layer of nearly confluent cells and media of similar refractive index to cytoplasm $\left(n=1.33\right.$ for media ${ }^{34}$ and $n=1.36$ for cytoplasm ${ }^{35}$ ), the beam (with a spot size of $3 \mathrm{~mm}$ ) is interacting with a layer of thousands of cells, where the membrane is just one object in the layer. Thus we conducted our analysis based on the volume of the membrane rather than the volume of the cell. While differences in cell size and shape may have effects on small-angle forward scattering $(0.5 \mathrm{deg}$ to $1.5 \mathrm{deg}$ ) of single cells, the cell membrane scattering effect in our cell layers would be minimal compared to other cellular organelles such as the nucleus. The membrane scattering volume is two orders of magnitude less than the nuclear scattering volume according to measurements cited in literature for murine macrophage membrane ${ }^{39,40}$ and nuclei. ${ }^{41}$ The large difference in scattering volume between these two cell components suggests that the cell membrane is least likely to contribute to the observed difference in scattering between M1 and M2 macrophage monolayers. The most likely candidates are smaller structures which are known to generate the greatest light scatteringnuclei, lysosomes, and mitochondria. $24,28,42$

The nucleus is a significant light scatterer in the cell ${ }^{21}$ and is known to be the dominant contributor to low-angle forward scattering at angles greater than $1.5 \mathrm{deg} .{ }^{23,24}$ Measurements of suspensions of isolated nuclei support this claim, as scattering from the isolated nuclei closely resembled those of intact cells at low angles. ${ }^{24}$ Despite these findings, studies by Mourant et al. on angular scattering distributions of $633 \mathrm{~nm}$ light between $3 \mathrm{deg}$ and $171 \mathrm{deg}$ have found that the dominant population of scatterers in rat embryo fibroblasts is smaller than the nucleus. $^{43}$

Furthermore, simulated scattering intensity calculations show that the difference in nuclear sizes needs to be quite large in order to explain the scattering differences alone. Due to the near confluence of the macrophages in our monolayers and the similar refractive index of the culture media and the cytoplasm of the cells $\left(n=1.33\right.$ for media ${ }^{34}$ and $n=1.36$ for cytoplasm ${ }^{35}$ ), variations in refractive index are small in the $x-y$ plane, allowing us to assume that the cell layer acts as an optical phase screen. ${ }^{44-50}$ In the phase screen, the wave field just above the cell layer, $a_{c}$, can be written as a function of the phase shift, ${ }^{50} \phi(x, y)$, experienced by the light propagating through the cells:

$a_{c}=\mathrm{e}^{j \phi(x, y)}$,

where $x$ and $y$ are the coordinates in the front focal plane of the lens. Since the variation in phase over the macrophage cell layer 

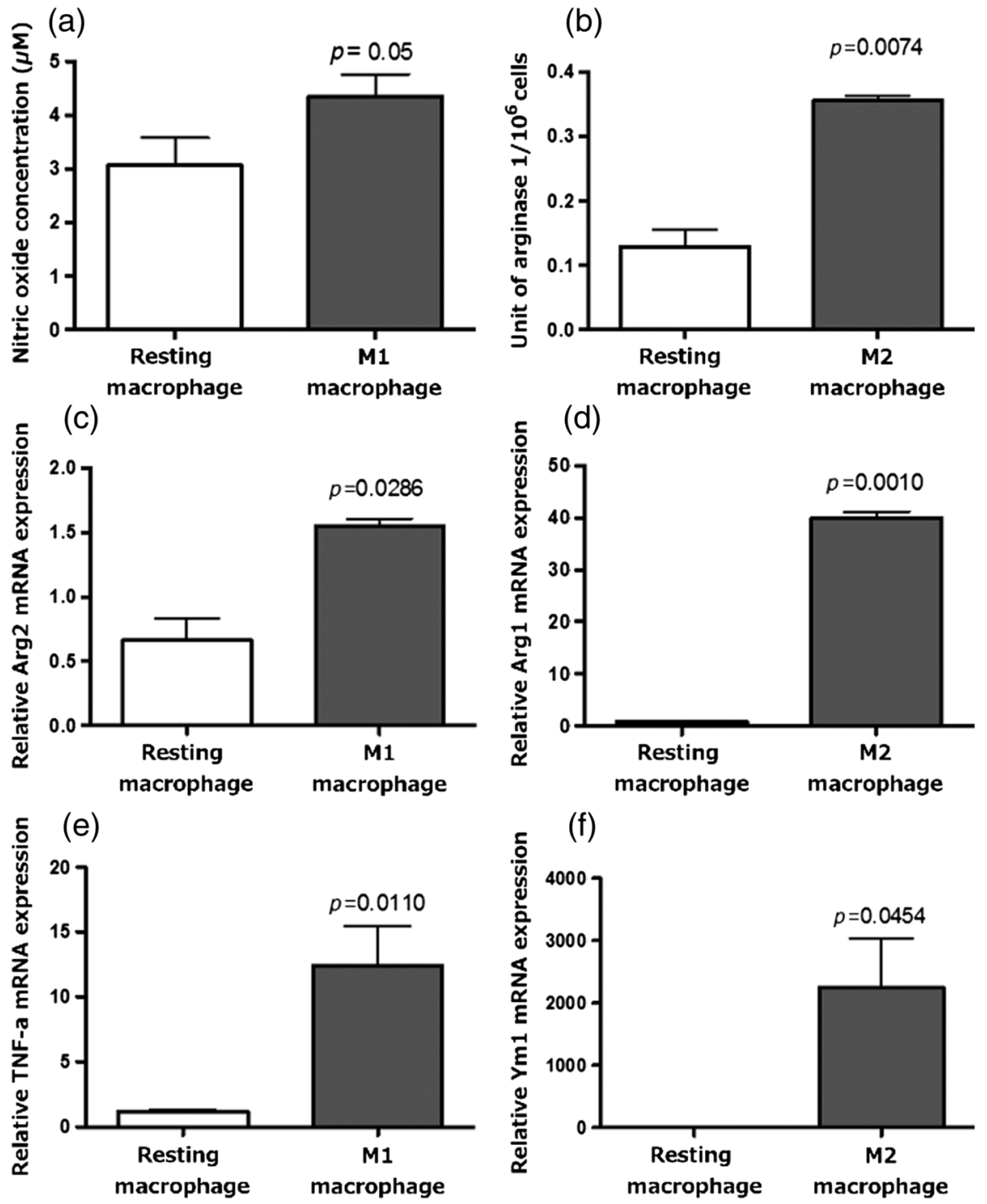

Fig. 4 Macrophage functional and molecular assays to confirm M1 and M2 polarization. (a) Macrophages treated with LPS and IFN- $\gamma$ produce significantly more nitric oxide than resting macrophages, consistent with the M1 phenotype. (b) Macrophages treated with IL-4 produce significantly more arginase 1 than resting macrophages, consistent with the M2 phenotype. (c, e) Macrophages treated with LPS and IFN- $\gamma$ have significantly higher expression of arginase 2 and tumor necrosis factor- $\alpha$ mRNA, respectively, than resting macrophages, consistent with the M1 phenotype. ( $d$, f) Macrophages treated with IL-4 have significantly higher expression of arginase 1 and Ym1 than resting macrophages, consistent with the M2 phenotype.

is small (less than $1 \mathrm{rad}$ ), we can use a Born approximation to rewrite the light amplitude as

$a_{c} \approx 1+j \phi(x, y)=1+j\left(\frac{t n_{\text {cells }}(x, y) 2 \pi}{\lambda}\right)$,

where $n_{\text {cells }}(x, y)$ is the spatial variation of the refractive index of the macrophages in the culture dish, $\lambda$ is the vacuum center wavelength of the light source, and $t$ is the thickness of the macrophage cell layer. ${ }^{50}$ While these approximations do not explicitly include internal interference within the cell organelles, they allow us to make calculations regarding the nuclear scattering effects. We observed that as the scattering angle increased, the M2 cells showed higher scattering intensity than did M1 cells [Fig. 3(b)]. At the upper end of our measurement range of $3.2 \mathrm{deg}$, the M2 scattering intensity was 1.6 times the M1 scattering intensity. Using the phase screen model, we compute that if the nuclear sizes differed, and for an average resting murine macrophage nuclear diameter of $6.50 \pm 0.23 \mu \mathrm{m},{ }^{41}$ the M1 cell nuclei diameter would have to be $86.1 \%$ greater than the M2 cell nuclei diameter on average to produce the observed intensity ratio at 3.2 deg. ${ }^{51,52}$ Similarly, taking the (M2) nucleus as a Mie scatterer with a diameter of 4 to $5.5 \mu \mathrm{m},{ }^{35}$ the larger (M1) nucleus would have had to be $69 \%$ or $37.3 \%$ larger, respectively, to explain the observed difference in scattering. This degree of nuclear diameter variation is greater than the increase in nuclear size observed even in cancer cells, ${ }^{53-}$ 55 and thus unlikely to exist between M1 and M2 macrophages. Further, it is known that the nuclear size of murine peritoneal macrophages does not differ between resting macrophages and M1 macrophages treated with LPS. ${ }^{56}$ Thus, we conclude that the observed difference in forward light scattering between 
M1 and M2 macrophages is likely not a nuclear effect alone, and an organelle other than the nucleus may be responsible for the observed light scattering differences.

Another strong light scatterer within cells is the lysosome. ${ }^{24,42,57}$ Lysosomes are roughly spherical or ovoid in shape and have a diameter of approximately 0.2 to $1 \mu \mathrm{m} .^{24,28,42}$ Due to their small size, lysosomes contribute strongly to high angle scattering ${ }^{24,28,42}$ (i.e., $90 \mathrm{deg}$ ). Marina et al. simultaneously measured the flow cytometry side-scatter of $785 \mathrm{~nm}$ light and organelle-specific fluorescence to demonstrate that lysosomes account for $20 \%$ to $30 \%$ of side-scatter by rat fibroblast cultures. ${ }^{28}$ A study by Wilson and Foster using a lysosomal ablation method to measure the lysosomal contribution to $633 \mathrm{~nm}$ light scattering by epithelial-mesenchymal transition 6 (EMT6) cells in culture reported that lysosomes account for $\sim 14 \%$ of total scattering between $7 \mathrm{deg}$ and $83 \mathrm{deg} .{ }^{42}$ However, scattering below $15 \mathrm{deg}$ was similar for control and lysosome-ablated cells, suggesting that the contribution of lysosomes to forward scattering in the range measured in our study was minimal. Further, there are no reported differences in the number, size, or distribution of lysosomes between M1 and M2 macrophages, making these organelles unlikely to be the cause of differential angular scattering distributions between our two macrophage subtypes.

Possibly the greatest contributors to total light scattering by cells are mitochondria, ${ }^{21,22,26,42}$ which range from 1 to $4 \mu \mathrm{m}$ in length and 0.3 to $0.7 \mu \mathrm{m}$ in diameter. ${ }^{24}$ For instance, Wilson et al. showed that $65 \%$ of total scattering by EMT6 cells in culture in the angular range of $5 \mathrm{deg}$ to $90 \mathrm{deg}$ was attributable to scatterers with sizes spanning 1 to $3 \mu \mathrm{m} .{ }^{23}$ A subsequent study by Wilson and Foster was in close agreement with these results and found that $77 \%$ of light scattered by EMT6 cells between $7 \mathrm{deg}$ and $83 \mathrm{deg}$ was due to a population of scatterers with mean size of $1.3 \mu \mathrm{m}$, consistent with mitochondria. ${ }^{42}$ The most dramatic example of mitochondrial scattering strength is from hepatocytes of the liver, in which the origin of light scattering is primarily due to this organelle. ${ }^{58}$ Hepatocytes are an extreme example, as mitochondria make up $28 \%$ of the cell volume, a significantly greater fraction of the cell volume than in other types of cells. ${ }^{24}$ Nonetheless, mitochondria are clearly significant scattering centers within the cell.

While measurements of isolated organelles ${ }^{24}$ and intact cells ${ }^{28}$ indicate that mitochondria are responsible for scattering at large angles, including flow cytometry side-scatter, mitochondria also contribute significant light scattering at small forward angles. Indeed, angular scattering distributions from isolated mitochondria demonstrate the highest scattering strength at the lowest angles measured by Wilson et al. $(5 \mathrm{deg})^{23}$ and Mourant et al. $(9 \mathrm{deg})^{24}$ (Fig. 5). Further, in Wilson's study, induced mitochondrial swelling caused a decrease in scattering by EMT6 cells compared to control cells with normal mitochondrial structure for angles between $5 \mathrm{deg}$ and $30 \mathrm{deg}$, while scattering at higher angles was similar between the two groups. While none of these previous studies were able to measure the contribution of mitochondrial light scattering to angles within the range of $1.6 \mathrm{deg}$ to $3.2 \mathrm{deg}$ examined in our study, we anticipate that mitochondria will contribute to scattering in this range. Known differences in the number of mitochondria between M1 and M2 macrophages ${ }^{30-}$ ${ }^{32}$ could account for the differences in light scattering observed in our study between these two macrophage subtypes, assuming that mitochondria act as discrete scattering centers.

In the current study, we found a difference in forward light scattering, suggesting that a difference in the angular

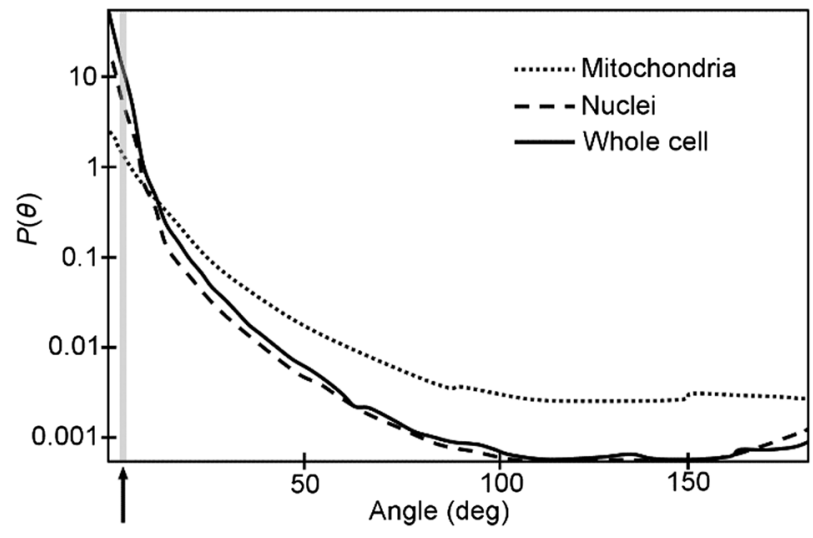

Fig. 5 Illustration of angular scattering distributions, $P(\theta)$, by cells, nuclei, and mitochondria (modified from Ref. 18). In the low angular range measured in our study (1.6 deg to $3.2 \mathrm{deg}$, gray bar and black arrow) the whole cell and nucleus are the dominant scatterers. However, mitochondria still make a substantial contribution in this angular range, greater than mitochondrial scattering at higher angles. Thus differences in the number of mitochondria between M1 and M2 macrophages would be expected to cause differences in light scattering between the two subtypes in the angular range measured in the current study. All curves were normalized in the original paper. ${ }^{18}$

dependence of the backscattered light could also exist between M1 and M2 macrophage cell layers as well. Many techniques using backscattered light for diagnostic information have been developed, which may be appropriate to take this backscatter measurement. For example, a scattering-angle-sensitive light scattering spectroscopy system has been developed by Backman et al. ${ }^{59}$ which uses a collimated, polarized incident beam to collect a two-dimensional map of the angular distribution of backscattered light. Angle-resolved low-coherence interferometry, developed by Wax et al., ${ }^{53}$ would also be appropriate. However, the results of the current study are only relevant for forward scattered light, which cannot be implemented directly as a clinical tool in intact tissues.

There are several limitations to the current study. We conducted our measurements in vitro with fully polarized M1 and M2 macrophages, while macrophages in vivo may exist in intermediate states. Second, the control of removing the mitochondria from both M1 and M2 macrophage subtypes was not performed to determine if the differences in light scattering could be eliminated. However, current techniques to remove mitochondria would make the macrophages nonviable, introducing additional variables, and thus would not be definitive. Third, we conducted our study with murine rather than human macrophages, although the increase in mitochondria in M2 murine and human macrophages compared to M1 are expected to be similar. In this study, we found a difference in forward scattering, suggesting that a difference in the angular dependence of backscatter could exist as well. However, additional studies are needed to evaluate angular backscattering. Finally, we conducted our analysis under the assumption that mitochondria are discrete scattering centers.

In conclusion, we demonstrated that M1 and M2 macrophages differ in their angular light scattering profiles between $1.6 \mathrm{deg}$ and $3.2 \mathrm{deg}$. Within this range, M2 scattered more light and had a slower signal drop-off than M1 macrophages, resulting in a significantly lower ratio of scattering intensity of $1.6 \mathrm{deg}: 3.2 \mathrm{deg}$ of M2 cultures compared to M1 cultures $(p<0.001)$. Finally, molecular and functional assays of M1 
and M2 macrophage cultures indicated successful polarization of the cells into these phenotypes. Future studies on optical differences between M1 and M2 macrophages may yield additional signatures-scattering or otherwise - that could be exploited to distinguish these subtypes and provide better disease diagnosis.

\section{Acknowledgments}

This work was supported by the Veterans Health Administration Merit Grant I01 BX000397; the Janey and Dolph Briscoe Center for Cardiovascular Research (San Antonio, Texas); the Clayton Foundation for Biomedical Research (Houston, Texas); the National Institutes of Health (T32 HL007446); an American Society for Laser, Medicine, and Surgery 2013-2014 Research Grant; and an American Heart Association Post-doctoral Award (13POST17080074).

\section{References}

1. J. P. Murray and T. A. Wynn, "Protective and pathogenic functions of macrophage subsets," Nat. Rev. Immunol. 11, 723-737 (2011).

2. F. O. Martinez et al., "Macrophage activation and polarization," Front. Biosci. 13, 453-461 (2008).

3. J. L. Stöger, P. Goossens, and M. P. J. de Winther, "Macrophage heterogeneity: relevance and functional implications in atherosclerosis," Curr. Vasc. Pharmacol. 8, 233-248 (2010).

4. A. Mantovani et al., "Macrophage polarization: tumor-associated macrophages as a paradigm for polarized M2 mononuclear phagocytes," Trends Immunol. 23, 549-555 (2002).

5. M. A. Bouhlel et al., "PPAR $\gamma$ activation primes human monocytes into alternative M2 macrophages with anti-inflammatory properties," Cell Metab. 6, 137-143 (2007).

6. G. Chinetti-Gbaguidi et al., "Human atherosclerotic plaque alternative macrophages display low cholesterol handling but high phagocytosis because of distinct activities of the PPAR $\gamma$ and $\operatorname{LXR} \alpha$ pathways," Circ. Res. 108, 985-995 (2011).

7. A. Sica et al., "Tumour-associated macrophages are a distinct M2 polarised population promoting tumour progression: potential targets of anti-cancer therapy," Eur. J. Cancer 42, 717-727 (2006).

8. G. Chinette-Gbaguidi and B. Staels, "Macrophage polarization in metabolic disorders: functions and regulation," Curr. Opin. Lipidol. 22, 365-372 (2011)

9. C. D. Mills, "M1 and M2 macrophages: oracles of health and disease," Crit. Rev. Immunol. 32, 463-488 (2012).

10. J. Khallou-Laschet et al., "Macrophage plasticity in experimental atherosclerosis," PLoS One 5, e8852 (2010).

11. M. Y. Donath and S. E. Shoelson, "Type 2 diabetes as an inflammatory disease," Nat. Rev. Immunol. 11, 98-107 (2011).

12. J. L. Stöger et al., "Distribution of macrophage polarization markers in human atherosclerosis," Atherosclerosis 225, 461-468 (2012).

13. S. Shaikh et al., "Macrophage subtypes in symptomatic carotid artery and femoral artery plaques," Eur. J. Vasc. Endovasc. 44, 491-497 (2012).

14. S. Fujisaka et al., "Regulatory mechanisms for adipose tissue M1 and M2 macrophages in diet-induced obese mice," Diabetes 58, 2574-2582 (2009).

15. B. Chance and L. Packer, "Light-scattering and absorption effects caused by addition of adenosine diphosphate to rat-heart-muscle sarcosomes," Biochem. J. 68, 295-297 (1958).

16. S. Izzard and H. Tedeschi, "Ion transport underlying metabolically controlled volume changes of isolated mitochondria," Proc. Natl. Acad. Soc. U. S. A. 67(2), 702-709 (1970)

17. L. Packer and A. L. Tappel, "Light scattering changes linked to oxidative phosphorylation in mitochondrial membrane fragments," J. Biol. Chem. 235(2), 525-530 (1960).

18. H. Tedeschi, J. M. James, and W. Anthony, "Photometric evidence for the osmotic behavior of rat liver microsomes," J. Cell Biol. 18, 503-513 (1963).
19. B. Chance and C. Lee, "Comparison of fluorescence probe and lightscattering readout of structural states of mitochondrial membrane fragments," FEBS Lett. 4, 181-184 (1969).

20. H. Tedeschi and D. L. Harris, "Some observations on the photometric estimation of mitochondrial volume," Biochim. Biophys. Acta 28, 392402 (1958)

21. N. N. Boustany, R. Drezek, and N. V. Thakor, "Calcium-induced alterations in mitochondrial morphology quantified in situ with optical scatter imaging," Biophys. J. 83, 1691-1700 (2002).

22. N. N. Boustany, S. C. Kuo, and N. V. Thakor, "Optical scatter imaging: subcellular morphometry in situ with Fourier filtering," Opt. Lett. 26, 1063-1065 (2001).

23. J. D. Wilson et al., "Light scattering from intact cells reports oxidativestress-induced mitochondrial swelling," Biophys. J. 88, 2929-2938 (2005).

24. J. R. Mourant et al., "Mechanisms of light scattering from biological cells relevant to noninvasive optical-tissue diagnostics," Appl. Opt. 37, 3586-3593 (1998).

25. B. Beauvoit et al., "Correlation between the light scattering and the mitochondrial content of normal tissues and transplantable rodent tumors," Anal. Biochem. 226, 167-174 (1995).

26. R. M. Pasternack, J. Y. Zheng, and N. N. Boustany, "Optical scatter changes at the onset of apoptosis are spatially associated with mitochondria," J. Biomed. Opt. 15, 040504 (2010).

27. K. J. Chalut et al., "Light scattering measurements of subcellular structure provide noninvasive early detection of chemotherapy-induced apoptosis" Cancer Res. 69, 1199-1204 (2009).

28. O. C. Marina, C. K. Sanders, and J. R. Mourant, "Correlating light scattering with internal cellular structures," Biomed. Opt. Express 3, 296-312 (2012).

29. J. W. Pyhtila, R. N. Graf, and A. Wax, "Determining nuclear morphology using an improved angle-resolved low coherence interferometry system," Opt. Express 11, 3473-3484 (2003).

30. J. Martinez et al., "The relationship between metabolism and the autophagy machinery during the innate immune response," Cell Metab. 17, 895-900 (2013).

31. D. Vats et al., "Oxidative metabolism and PGC- $1 \beta$ attenuate macrophage-mediated inflammation," Cell Metab. 4, 13-24 (2006).

32. S. Tavakoli et al., "Bioenergetic profiles diverge during macrophage polarization: implications for the interpretation of 18F-FDG PET imaging of atherosclerosis," J. Nucl. Med. 54, 1661-1667 (2013).

33. Y. Sowa, B. C. Steel, and R. M. Berry, "A simple backscattering microscope for fast tracking of biological molecules," Rev. Sci. Instrum. 81, 113704 (2010).

34. N. Lue et al., "Live cell refractometry using microfluidic devices," Opt. Lett. 31, 2759-2761 (2006).

35. L. T. Perelman et al., "Observation of periodic fine structure in reflectance from biological tissue: a new technique for measuring nuclear size distribution," Phys. Rev. Lett. 80, 627-630 (1998).

36. J. D. Gaskill, "The propagation and diffraction of optical wave fields," in Linear Systems, Fourier Transforms, and Optics, pp. 417, John Wiley \& Sons, Inc., Toronto, California (1978).

37. P. Shivshankar et al., "Caveolin-1 deficiency protects from pulmonary fibrosis by modulating epithelial cell senescence in mice," Am. J. Respir. Cell. Mol. Biol. 47, 28-36 (2012).

38. H. Wang et al., "Altered macrophage phenotype transition impairs skeletal muscle regeneration," Am. J. Pathol. 184, 1167-1184 (2014).

39. K. Mitra et al., "Modulation of the bilayer thickness of exocytic pathway membranes by membrane proteins rather than cholesterol," Proc. Natl. Acad. Soc. U. S. A. 101, 4083-4088 (2004).

40. F. Krombach et al., "Cell size of alveolar macrophages: an interspecies comparison," Environ. Health Prospect. 105, 1261-1263 (1997).

41. K. J. Chalut et al., "Label-free, high-throughput measurements of dynamic changes in cell nuclei using angle-resolved low coherence interferometry," Biophys. J. 94, 4948-4956 (2008).

42. J. D. Wilson and T. H. Foster, "Characterization of lysosomal contribution to whole-cell light scattering by organelle ablation," J. Biomed. Opt. 12, 030503 (2007).

43. J. R. Mourant, T. M. Johnson, and J. P. Freyer, "Characterizing mammalian cells and cell phantoms by polarized backscattering fiberoptic measurements," Appl. Opt. 40, 5114-5123 (2001). 
44. R. Wu and L. Huang, "Scattered field calculation in heterogeneous media using a phase-screen propagator," Geophysics 50, 1289-1292 (1992).

45. V. V. Tuchin et al., "Light propagation in tissues with controlled optical properties," J. Biomed. Opt. 2, 401-417 (1997).

46. E. Jakeman and J. G. McWhirter, "Fluctuations in radiation scattered into the Fresnel region by a random-phase screen in uniform motion," J. Phys. A: Math. Gen. 9, 785 (1976).

47. T. T. Wu, J. Y. Qu, and M. Xu, "Unified Mie and fractal scattering by biological cells and subcellular structures," Opt. Lett. 32, 2324-2326 (2007).

48. M. Xu, "Scattering-phase theorem: anomalous diffraction by forwardpeaked scattering media," Opt. Express 19, 21643-21651 (2011).

49. B. DeAngelo et al., "Determination of the scattering coefficient, the reduced scattering coefficient, and the anisotropy factor of tissue with differential interference contrast microscopy," Proc. SPIE 8230, 82300I (2012).

50. J. W. Goodman, "Spatial filtering and optical information processing," in Introduction to Fourier Optics, pp. 145, McGraw-Hill Companies, Inc., New York (1988).

51. J. D. Gaskill, "Special functions," in Linear Systems, Fourier Transforms, and Optics, pp. 72, John Wiley \& Sons, Inc., Toronto, California (1978).

52. F. L. Pedrotti, L.S. Pedrotti, and L.M. Pedrotti, "Fraunhofer diffraction," in Introduction to Optics, pp. 277, Pearson Prentice Hall, New Jersey (2007).

53. A. Wax et al., "In situ detection of neoplastic transformation and chemopreventive effects in rat esophagus epithelium using angle-resolved lowcoherence interferometry," Cancer Res. 63, 3556-3559 (2003).

54. N. Terry et al., "Detection of intestinal dysplasia using angle-resolved low coherence interferometry," J. Biomed. Opt. 16, 106002 (2011).

55. K. J. Pienta and D. S. Coffey, "Correlation of nuclear morphometry with progression of breast cancer," Cancer 68, 2012-2016 (1991).

56. Z. A. Cohn, J. G. Hirsch, and M. E. Fedorko, "The in vitro differentiation of mononuclear phagocytes," J. Exp. Med. 123, 747-756 (1966).

57. M. Daigneault et al., "The identification of markers of macrophage differentiation in PMA-stimulated THP-1 cells and monocyte-derived macrophages," PLoS One 5, e8668 (2010).

58. B. Beauvoit, T. Kitai, and B. Chance, "Contribution of the mitochondrial compartment to the optical properties of rat liver: a theoretical and practical approach,” Biophys. J. 67, 2501-2510 (1994).

59. V. Backman et al., "Imaging and measurement of cell structure and organization with submicron accuracy using light scattering spectroscopy," Proc. SPIE 4613, 101-110 (2002).

David L. Halaney received his BS degree in biology from Trinity University, San Antonio, Texas, USA, in 2011. Currently, he is employed as a research assistant at the University of Texas Health Science Center in San Antonio.
Aydin Zahedivash is pursuing a BS degree in biomedical engineering from the University of Texas at Austin Cockrell School of Engineering. Currently, he works with the UT Biomedical Engineering Laser Lab to help implement light-based technologies for diagnostic and therapeutic potential.

Jennifer E. Phipps received her BS degree in electrical engineering from the University of Washington and her PhD in biomedical engineering from the University of California-Davis. Her research focuses on optical imaging techniques for improving cardiovascular disease diagnostics.

Tianyi Wang received his $\mathrm{PhD}$ in biomedical engineering from University of Texas at Austin in 2012. Currently, he is a research associate at the University of Texas at Austin, developing a multimodal OCT system for enhanced characterization of atherosclerotic lesions.

Jordan Dwelle received his $\mathrm{PhD}$ in biomedical engineering from the University of Texas at Austin in 2011. His research focus was polarization sensitive optical coherence detection for early glaucoma detection. Currently, he works at JP3 Measurement in Austin, Texas, USA.

Claude Jourdan Le Saux obtained her PhD in the Institute Pasteur of Lyon on cell and molecular biology in 1996. Since her PhD, her research focus has been fibrosis and the mechanisms that trigger fibrosis, including inflammation and more specifically the activation of the macrophages.

Reto Asmis received his PhD in biochemistry from the University of Fribourg, Switzerland. He is a professor, senior associate dean, and industry consultant with more than 20 years of experience establishing, leading, and growing innovative life sciences programs in the United States and Europe. At the University of Texas Health Science Center at San Antonio (UTHSCSA), he directs federally funded basic, biomedical, and translational interdisciplinary research programs in cardiovascular biology, vascular imaging, and nutrition.

Thomas E. Milner received his $\mathrm{PhD}$ in optical sciences from the University of Arizona in 1991. After working as a research faculty member at the Beckman Laser Institute and Medical Clinic (1992 to 1997), he joined the engineering faculty at the University of Texas in Austin (1998), where he currently resides. His research is directed toward the development of novel optical tomographic imaging modalities and laser surgical procedures for the diagnosis and treatment of human diseases.

Marc D. Feldman received his MD degree from the University of Pennsylvania School of Medicine in 1981. Since 1998, he has served as faculty at the University of Texas Health Science Center at San Antonio, where he practices interventional cardiology and leads a research lab focusing on the use of optical coherence tomography to predict subsequent rupture of atherosclerotic plaques and myocardial infarction. 